\title{
Multi Operated Virtual Power Plant in Smart Grid
}

\author{
Yevhen Fediv, Olha Sivakova, Mykhailo Korchak* \\ Lviv Polytechnic National University, Department of Electric Power Engineering and Control Systems, Institute of Power \\ Engineering and Control Systems, Lviv, 79012, Ukraine
}

\begin{tabular}{l} 
A R T I C L E I N F O \\
\hline Article history: \\
Received: 23 August, 2020 \\
Accepted: 14 October, 2020 \\
Online: 10 November, 2020 \\
\hline Keywords: \\
Virtual power plant \\
Smart grid \\
AC voltage controller \\
GTO thyristor \\
Reactive power \\
IoT
\end{tabular}

A B S T R A C T
In order to balance the power in intelligent distribution networks (Smart Grid or Microgrid),
it is proposed to organize a «multi operated virtual power plant». The resources of active and
reactive power for which can be obtained using an AC voltage controller with a phase-angle
control for regulation of operating modes of the ohmic load of consumers, for example,
distributed systems of electric space heating or electric water heating, etc. A method is
proposed and the results of the analysis of the phase-angle control modes by gate turn off
(GTO) thyristors of the AC voltage regulator, which provides the generation of virtual
reactive power by consumers of active power, are presented. The process equipment of such
a virtual power plant is fully suitable both for the dynamic production of a virtual resource of
active power to balance, for example, the power of dynamic distributed renewable energy
sources (virtual power plant (VPP) mode), and to regulate reactive power to ensure adequate
voltage levels and increase stock stability of operation of electric load units (virtual reactive
power plant (VRPP) mode). References 24, figures 8.

\section{Introduction}

This article is an extension and continuation of the work presented in the materials of the IEEE conference [1], which shows the possibility of power electronics to obtain a resource of reactive power from consumers-regulators with an active nature of consumption. Improving the efficiency of power supply, development in the direction of intellectualization according to the concept of Smart Grid is based on modern advances in power electronics [2-4].

In addition to balancing active power with frequency stabilization, an important operational task in Smart Grid systems is to ensure proper voltage levels in power grid units, increase the stability margin of power generation and consumption systems, and optimize operating costs in the transportation and distribution of electricity.

The basis for solving these problems is to ensure the balance of reactive power in the system, the availability of appropriate resources and the ability to quickly involve them at different levels of transmission, conversion and distribution of electricity $[5,6]$. In the Smart Grid concept, the problem of reactive power compensation is highlighted as key. Different methods of reactive power compensation are considered in $[7,8]$.

"Corresponding author: Mykhailo Korchak., misha.korkirov@gmail.com $+380981712257$
The universal and most efficient centralized source of reactive power at present is the Static Compensator (Statcom) device [9], which is primarily intended for flexible power transmission systems or powerful industrial power supply systems.

Problems of using Statcom in low-voltage networks and their interaction with distributed energy sources are considered in articles $[10,11]$, where special attention is paid to the use of photovoltaic inverters as reactive power sources.

In [12-14], the use of static compensator D-STATCOM for unbalanced radial power distribution systems is investigated in order to ensure voltage stability within acceptable limits. This review of known works addresses issues related to the optimal choice of power, control methods, installation location, etc. of reactive power sources as physical objects installed by the power supplier. However, currently in the Smart Grid an important role is given to virtual energy sources

In the classical sense, a VPP is a distributed power plant that combines the resources of active power generation from distributed energy resources (DER) as a reserve at the disposal of transmission and distribution operators [15]. VPPs in Microgrid are able to increase resource efficiency and maintain the necessary balance of generation and consumption in the power system. Distributed renewables and drives in VPPs are not virtual but real 
resources. In fact, virtual (feigned) generation of electricity will be equivalent to refusing or limiting its consumption. For example, by integrating water heating or electric heating systems into a grid and remotely controlling them with electricity consumption, you can align the active power consumption schedule at a particular distribution network node without significant consequences for household or industrial consumers themselves.

Another possibility is the participation of customers in virtual power plants to provide reactive energy. [16-20] discusses the provision of reactive power services from DER using a market mechanism and a plan to convert Microgrid into VPP, which is beneficial for consumers, prosumers and utility operators.

Involving distributed sources to solve the problem of reactive power balancing, by analogy with a virtual power plant, can be combined into virtual reactive power sources. In [21, 22] the problem of reactive power is not allocated separately but included in the list of tasks for the organization of VPP. [23] presents the concept of a dynamic VRPP as an ancillary service to reasonably combine decentralized power distribution sources into a coordinated pool to provide reactive power for the needs of power transmission system operators. Common in the known concepts is the involvement in the VRPP real distributed sources of reactive power.

\section{Purpose and task of the research}

The aim of the work is to study the problem of balancing active and reactive power in distribution networks with the involvement of consumers' resources in the form of integrated virtual power plant (VPP-VRPP), where reactive power resource is obtained by regulating the receivers used for VPP. This is especially true for facility power systems that are not encouraged to install their own sources to compensate for reactive power.

The ranges of complex regulation by means of power electronics of consumers with active character of loading for reception of a virtual resource of active and reactive power by regulation according to needs of operators of power supply systems are substantiated in the work.

\section{Finding a solution to the problem}

Figure 1 shows the equivalent circuit diagram of one phase VPP-VRPP, which shows the AC voltage regulator, which includes a source of sinusoidal voltage with internal inductance, a block of power dual-operation (GTO) thyristors VS1 and VS2, a limiting diode suppression diode) $\mathrm{VD}$, as well as resistive resistance $\mathrm{R}$ of water heater or electric heating.

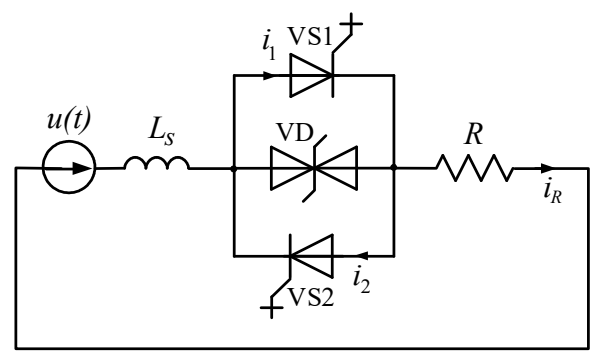

Figure 1: Equivalent power supply circuit of the heater
To protect against the effects of rigid switching of thyristors inductive overvoltage emissions in the interval between the closing of the thyristors, a two-way TVS diode VD is designed.

To obtain the energy characteristics in the steady state VPPVRPP, we assume that the thyristors VS1, VS2 are ideal switches, not taking into account the diode VD, inductance $L_{s}=0$, and voltage of the power supply system is sinusoidal as shown in (1)

$$
u(t)=U_{m} \sin \left(\omega t+\varphi_{u}\right)
$$

where $U_{m}$ is power supply voltage amplitude; $\varphi_{u}$ is the initial phase of the supply voltage; $\omega$ is angular velocity.

To obtain the power in the circuit of the resistor, controlled by key semiconductor elements, we have to analyze two characteristic modes.

\subsection{Mode of consumption of virtual reactive power from the power supply network.}

This mode is characteristic for traditional single-operation (semi-controlled) thyristors or SCR (Semiconductor Controlled Rectifier). For its realization thyristors VS1 and VS2 is being opened by the controlling impulse,_with a delay of an angle $\alpha$ relative to the natural angle of their entry into operation $\alpha_{0}=0 \pm \pi k$, where $k=0,1,2 \ldots$, and closing them at the natural time of closing $\beta_{0}=\pi+\pi k$ (Fig. 2).



Figure 2: VRPP current and voltage time diagrams in the mode of reactive power consumption

As a result, we obtained the shift of the main harmonic of the source current $i_{(1) R}$ towards the delay relative to the voltage of the grid. It is equivalent to the consumption of virtual reactive power by electrical circuit, in which reactive elements are not presented.

It should be noted that the value of the reference times of the thyristor control angles (natural value of the angles of opening and closing of the thyristors) are characteristic of the purely active nature of the circuits with key elements.

To evaluate the energy effect of the above method of regulating the operation of the electric receiver, we decompose the current $i_{R}$ in the Fourier series. $n$-harmonic component of the relative current is descripted in expression (2)

$$
i_{n}(\omega t)=I_{n m *} \cdot \sin \left(n \omega t+\varphi_{n}\right)
$$

can be obtained as (3) 


$$
I_{n m *}=\frac{I_{n m}}{I_{b}}=\sqrt{A_{n^{*}}^{2}+B_{n^{*}}^{2}}
$$

where $I_{b}$ is the current amplitude in the mode of full conductivity of thyristors according to (4)

$$
I_{b}=I_{m, R}=U_{m} / R
$$

$A_{n *}$ and $B_{n *}$ are the coefficients for the decomposing to the Fourier series is written using (5) and (6):

$$
\begin{aligned}
A_{n^{*}} & =\frac{1}{\pi} \int_{\alpha}^{\pi} i_{R}(\omega t) \cos n \omega t d(\omega t)+\frac{1}{\pi} \int_{\pi+\alpha}^{2 \pi} i_{R}(\omega t) \cos n \omega t d(\omega t) \\
= & \frac{1}{2 \pi}\left\{\frac{\cos (n \pi)+\cos [(1-n) \alpha]-\cos (2 n \pi)+\cos [(1-n)(\pi+\alpha)]}{1-n}\right. \\
+ & \left.\frac{\cos (n \pi)+\cos [(1+n) \alpha]-\cos (2 n \pi)+\cos [(1+n)(\pi+\alpha)]}{1+n}\right\} \\
& \frac{1}{\pi} \int_{\pi+\alpha}^{2 \pi} i_{R} \frac{1}{\pi} \int_{\alpha}^{\pi} i_{R}(\omega t) \sin n \omega t d(\omega t)+ \\
= & \frac{1}{2 \pi}\left\{\frac{-\sin [(1-n) \alpha]-\sin [(1-n)(\pi+\alpha)]}{1-n}\right. \\
& \left.+\frac{\sin [(1+n) \alpha]+\sin [(1+n)(\pi+\alpha)]}{1+n}\right\}
\end{aligned}
$$

For the $1^{\text {st }}$ harmonic ( $\left.n=1\right)$ obtained equations (7) and (8):

$$
\begin{aligned}
& A_{1 *}=\frac{1}{2 \pi}[\cos (2 \alpha)-1] \\
& B_{1 *}=\frac{1}{2 \pi}[\sin (2 \alpha)+2(\pi-\alpha)]
\end{aligned}
$$

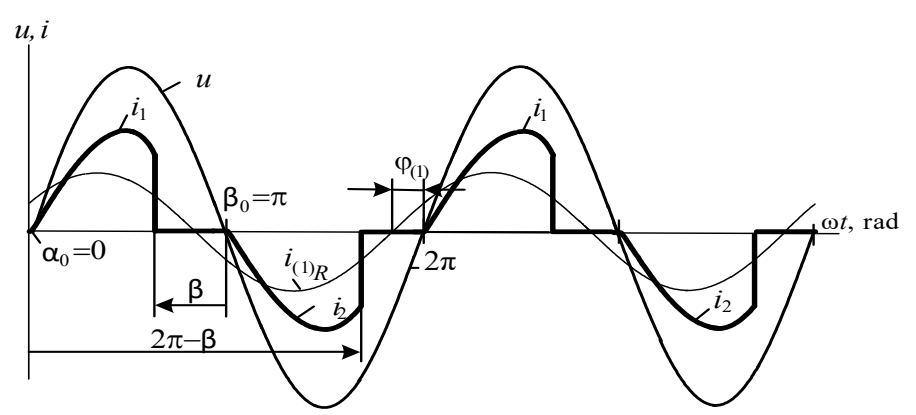

Figure 3: VRPP current and voltage time diagrams in the mode of reactive power generating

3.2. Mode of virtual reactive power generation in the power supply network.

This mode is dual to the previous one. For its realization thyristors VS1 and VS2 is being opened in the natural moments of their entry into operation $\alpha_{0}=0+\pi k$, where $k=0,1,2 \ldots$, and www.astesj.com closing with an advance on the angle $\beta$ relative to the natural angle $\beta_{0}$ of closure (Fig. 3). As a result, we obtained the shift of the main harmonic of the source current $i_{(1) R}$ in the direction of advance relative to the grid voltage, which is equivalent to generation of reactive power by resistance into the power supply system.

The formulas obtained by the authors for the decomposition coefficients in the Fourier series of the resistor current take the form (9) and (10).

$$
\begin{aligned}
& A_{n^{*}}=\frac{1}{\pi} \int_{0}^{\pi-\beta} i_{R}(\omega t) \cos n \omega t d(\omega t) \\
& +\frac{1}{\pi} \int_{\pi}^{2 \pi-\beta} i_{R}(\omega t) \cos n \omega t d(\omega t) \\
& =\frac{1}{2 \pi}\left\{\frac{1-\cos [(1-n)(\pi-\beta)]-\cos [(1-n)(2-\beta)]-\cos (n \pi)}{1-n}\right. \\
& \left.+\frac{1-\cos [(1+n)(\pi-\beta)]-\cos [(1+n)(2 \pi-\beta)]-\cos (n \pi)}{1+n}\right\} \\
& B_{n^{*}}=\frac{1}{\pi} \int_{0}^{\pi-\beta} i_{R}(\omega t) \sin n \omega t d(\omega t) \\
& +\frac{1}{\pi} \int_{\pi}^{2 \pi-\beta} i_{R}(\omega t) \sin n \omega t d(\omega t) \\
& =\frac{1}{2 \pi}\left\{\frac{\sin [(1-n)(\pi-\beta)]+\sin [(1-n)(2 \pi-\beta)]}{1-n}\right. \\
& \left.-\frac{\sin [(1+n)(\pi-\beta)]+\sin [(1+n)(2 \pi+\beta)]}{1+n}\right\}
\end{aligned}
$$

For the $1^{\text {st }}$ harmonic $(n=1)$ we obtained equations (11) and (12):

$$
\begin{aligned}
& A_{1 *}=\frac{1}{2 \pi}[1-\cos (2 \beta)] \\
& B_{1 *}=\frac{1}{2 \pi}[2(\pi-\beta)+\sin (2 \beta)]
\end{aligned}
$$

\section{Generalization of the results of the analysis of control regimes}

In both of the above modes of thyristor control with a change in the value of the control angles and there is a simultaneous change in both active and reactive power, the value of which can be calculated by expressions (13) and (14).

$$
\begin{gathered}
P_{1 *}=\sqrt{A_{1 *}^{2}+B_{1 *}^{2}} \cos \left(\varphi_{1 u}-\varphi_{1 i}\right)=I_{1 m *} \cos \varphi_{1} \\
Q_{1 *}=I_{m 1} \sin \varphi_{1}
\end{gathered}
$$

where, $P_{1 *}, Q_{1 *}$ 


$$
\sqrt{A_{1 *}^{2}+B_{1 *}^{2}}=I_{1 m *}-\text { the amplitude of }
$$

the fundamental harmonic of the current, $\varphi_{1}=\arctan \left(B_{1} / A_{1}\right)-$ the initial phase of the fundamental harmonic of the current, and $\varphi_{1 u}=0$.

Figure 4 shows the results of the calculation of VPP-VRPP control characteristics by reactive power. The maximum generation of reactive power is $\left|Q_{1 *}\right| \approx 0,32$ p.u. different signs are reached at the control angles of the boards $\alpha=\beta=\pi / 2$. In case of regulating the VPP-VRPP by changing the opening angles $\alpha=$ var of thyristors and constant closing angles $\beta=$ const $=0$

from the mains consumes excess reactive bias power $\left(Q_{1}>0\right)$, and by constant angles of thyristor opening $(\alpha=$ const $=0)$ and adjusting the angle $\beta=$ var change we get the mode of generating reactive bias power to the mains $\left(Q_{1}<0\right)$.

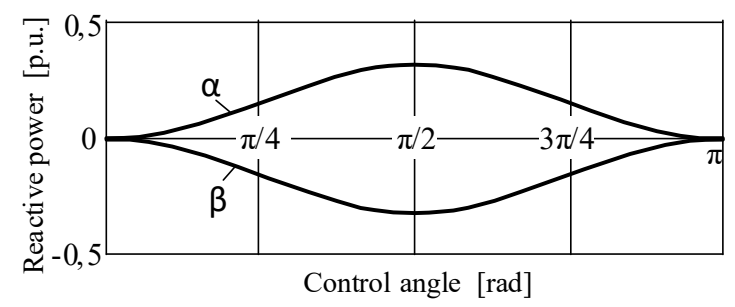

Figure 4: VPP-VRPP control characteristics by reactive power

The obtained characteristics are symmetric with respect to the angle $\alpha=\beta=\pi / 2$, but in the mode of reactive power control the preference should be given to the range of angles $\alpha=\beta=0 \ldots \pi / 2$ of regulation of the boards, because in the range of angles $\alpha=\beta=\pi / 2 \ldots \pi$ we get a significant harmonic current distortion (Fig. 5)

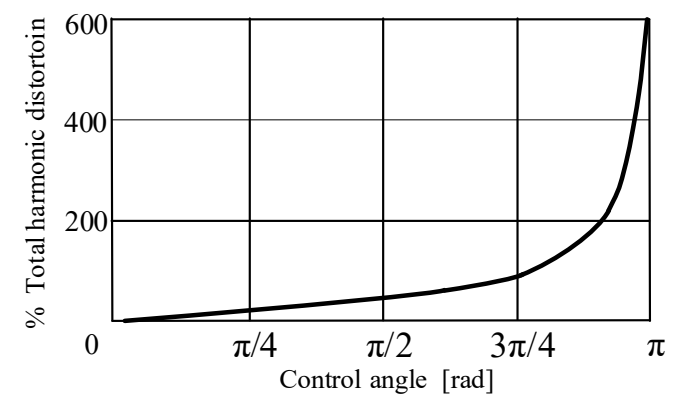

Figure 5: Coefficient of harmonic current distortion

Calculated by expression (15)

$$
T H D_{I}(\%)=\frac{I_{\text {dis }}}{I_{1}} 100=\frac{\sqrt{\sum_{n \neq 1} I_{n}^{2}}}{I_{1}} 100,
$$

The value of the harmonic distortion coefficients of the current consumed from the mains for the above-recommended range of adjustment of the control angles is $T H D_{I}=(0 \ldots 36,6) \%$. For the mode of full conductivity of thyristors, there is no distortion, and for the modes $\alpha=\beta=\pi / 2$ the harmonic distortion of VPP-VRPP current is $T H D_{I} \approx 36,6 \%$.

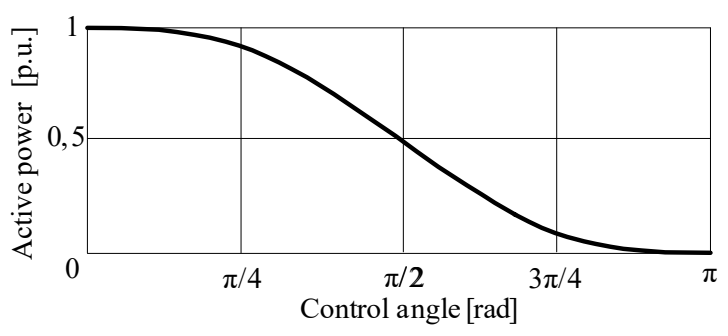

Figure 6: VPP-VRPP control characteristic by active power

The change in the reactive power of the VPP-VRPP in the process of regulation is accompanied by a simultaneous change in the active power consumed by the electric receiver (Fig. 6).

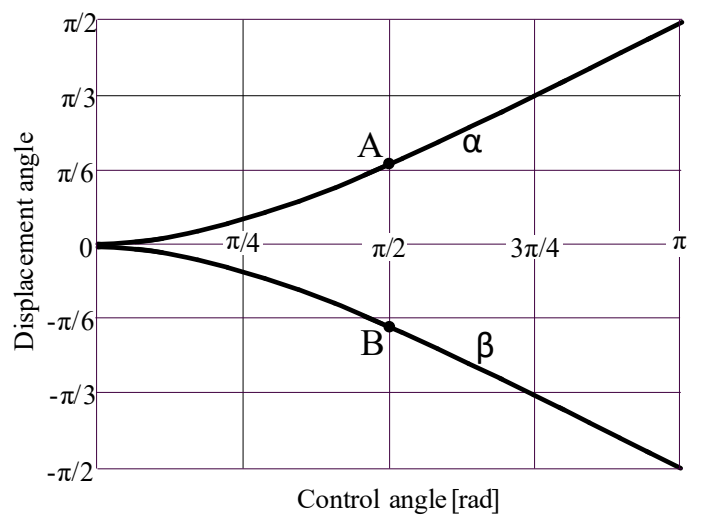

Figure 7: Displacement angle for two ways of regulation (modes) VRPP

As you can see from Fig. 4 and fig. 6, in the process of regulation, with increasing value of reactive power, the value of active power decreases, which generally contributes to the tasks to be solved by virtual power plants, the control effect on the active $d P / d \alpha<0, d P / d \beta<0$ and reactive $d Q / d \alpha>0, d Q / d \beta>0$ power of the resistive electrical receiver is different in signs.

We obtained modes in which the generation of reactive power to balance it in the load center is accompanied by a simultaneous decrease in active power consumption, which contributes to both the normalization of the voltage level (VRPP task) and active power balancing (VPP task).

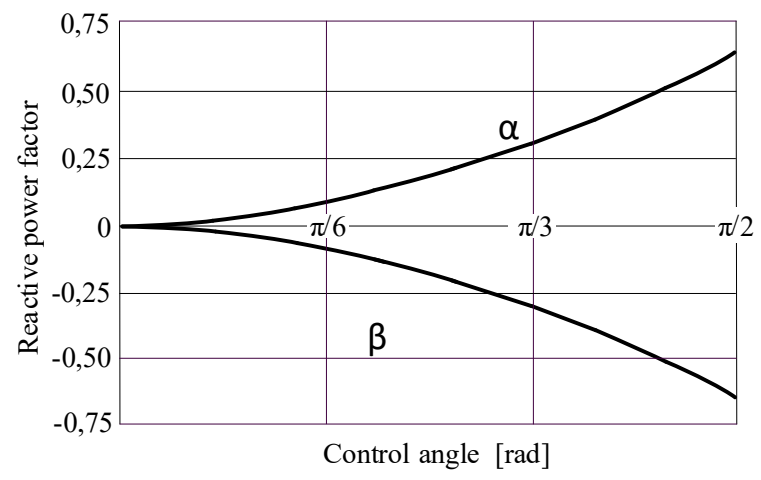

Figure 8: Reactive displacement power factor 
In the mode of maximum reactive power $\alpha=\beta=\pi / 2$, the value of the displacement angle of the main harmonic of the current is $\varphi_{1} \approx \pm 32,5^{\circ} \quad$ (points $\mathrm{A}$ and $\mathrm{B}$ on the corresponding characteristics of Fig. 7), and the coefficient of reactive displacement power (Fig. 8) $\operatorname{tg} \varphi_{1}= \pm 0,64$.

\section{Conclusions}

The article considers a comprehensive approach to solving the problems of active and reactive power balances in Microgrid networks, using hidden (virtual) resources, which are organized in the form of a virtual active-reactive power plant (VPP-VRPP). Using distributed resources of consumers and their active role in the operation of Microgrid.

Resources of active and reactive power are received from one source - ohmic receivers of consumption with involvement of means of power electronics as regulating elements.

By feeding the ohmic load from voltage regulators with GTO thyristors, the distribution network operator, if necessary, reduces the consumption of active power and adjusts the value and sign (consumption from grid or grid generation) of reactive power.

The range of change of control angles of thyristors of a voltage regulator is chosen for reasons of reduction of negative influence of nonlinear electric loading on quality of the electric power in Microgrid.

The use of integrated multi-controlled VPP-VRPP can be recommended for damping transients and ensuring the stability of Microgrid operation in modes with frequency and voltage value close to critical, to prevent avalanche-like changes.

\section{Conflict of Interest}

The authors declare no conflict of interest.

\section{Acknowledgment}

The authors thank both the authorities of the Lviv Polytechnic National University especially leadership of the Institute of Power Engineering and Control Systems for their support during the preparation of this paper. The authors also thank the anonymous reviewers, whose comments significantly improved the content of the paper.

\section{References}

[1] Y. Fediv, O. Sivakova, M. Korchak, "Model of Virtual Source of Reactive Power for Smart Electrical Supply Systems," in 2019 IEEE 20th International Conference on Computational Problems of Electrical Engineering (CPEE), 1-4, 2019, doi:10.1109/CPEE47179.2019.8949159.

[2] G. Benysek, M.P. Kazmierkowski, J.Popczyk, R. Strzelecki, "Power electronic systems as a crucial part of Smart Grid infrastructure - a survey," Bulletin of the polish academy of sciences technical sciences, 59(4), 2011, doi:10.2478/v10175-011-0058-2.

[3] B.K. Bose, "Power Electronics in Renewable Energy Systems and Smart Grid: Technology and Applications," Wiley-IEEE Press, 2019.

[4] B. K. Bose, "Power Electronics in Smart Grid and Renewable Energy Systems," in Proceedings of the IEEE, 105(11), 2007-2010, 2017, doi:10.1109/JPROC.2017.2752538.

[5] J. Ali, S. Massucco, G. Petretto, "Reactive power provision to TSO/DSO by aggregators and conventional generators," 2017 IEEE International Conference on Smart Grid Communications (SmartGridComm), 486-491, 2017, doi:10.1109/SmartGridComm.2017.8340740.

[6] K. Wang, M.L. Crow, "Electricity Transmission, Distribution and Storage Systems," Woodhead Publishing Series in Energy, 2013.
[7] J. Ali, S. Massucco, F. Silvestro, "STATCOM application for voltage profiling of a distribution grid with high penetration of distributed energy resources," Mediterranean Conference on Power Generation, Transmission, Distribution and Energy Conversion (MEDPOWER 2018), 1-7, 2018, doi:10.1049/cp.2018.1883.

[8] J. Ali, S. Massucco, F. Silvestro, "Aggregation Strategy for Reactive Power Compensation Techniques-Validation," Energies 2019, 1-13, 2019, doi:10.3390/en12112047.

[9] Q. Wang, B. Wang, W. Xu, J. Xu, "Research on STATCOM for reactive power flow control and voltage stability in microgrid," 2018 13th IEEE Conference on Industrial Electronics and Applications (ICIEA), Wuhan, 2474-2479, 2018, doi:10.1109/ICIEA.2018.8398126.

[10] S. Hasan, R. Luthander, J. de Santiago, "Reactive Power Control for LV Distribution Networks Voltage Management," in 2018 IEEE PES Innovative Smart Grid Technologies Conference Europe (ISGT-Europe), 1-6, 2018, doi:10.1109/ISGTEurope.2018.8571817.

[11] D. Sailaja, V.B. Kumar, "A Voltage Regulator for Power Quality Improvement in Low-Voltage Distribution Grids," Journal of Applied Science and Computations, 5(7), 461-468, 2018, DOI:10.1109/TPEL.2017.2693239.

[12] A.R. Guptaa, A. Kumar, "Impact of D-STATCOM placement on improving the reactive loading capability of unbalanced radial distribution system," Procedia Technology 25, 676-683, 2016, doi: 10.1016/j.protcy.2016.08.170.

[13] W. Guoyou, Z. Xilin, "Study on hybrid reactive power compensation in lowvoltage distribution system," International Conference on Energy and Environmental Protection (ICEEP 2016), 618-622, 2016, doi:10.2991/iceep-16.2016.106.

[14] L. E. Christian, L. M. Putranto, S.P. Hadi, "Design of Microgrid with Distribution Static Synchronous Compensator (D-STATCOM) for Regulating the Voltage Fluctuation," 2019 IEEE 7th International Conference on Smart Energy Grid Engineering (SEGE), 48-52, 2019, doi:0.1109/SEGE.2019.8859860

[15] M.M. Othman, Y.G. Hegazy, A.Y. Abdelaziz, "A Review of Virtual power plant Definitions, Components, Framework and Optimization," International Electrical Engineering Journal (IEEJ), 2010-2024, 6(9), 2015.

[16] M. Korchak, O. Sivakova, Y. Fediv, «Virtual source of reactive power in electricity supply systems of household consumers,» Energy Engineering and Control Systems, 9-14, 5(1), 2019 doi:10.23939/jeecs2019.01.009.

[17] D. Pudjianto, P. Djapic, G. Strbac, B. Stojkovska, A. R. Ahmadi, I. Martinez, "Integration of distributed reactive power sources through Virtual Power Plant to provide voltage control to transmission network," 25thInternational Conference on Electricity Distribution (CIRED-2019), 1-5, (2055), 2019. http://dx.doi.org/10.34890/933.

[18] J. Ali, S. Massucco, F. Silvestro, A. Vinci, «Participation of Customers to Virtual Power Plants for Reactive Power Provision,» Conference: 2018 53rd International Universities Power Engineering Conference (UPEC), 1-6, 2018, doi:10.1109/UPEC.2018.8541978.

[19] K. L. Anaya, M. G. Pollitt, "Reactive Power Procurement: Lessons from Three Leading Countries," Cambridge Working Papers in Economics, 2018

[20] J. Naughton, H. Wang, S. Riaz, M. Cantoni, P. Mancarella, "Optimization of Multi-Energy Virtual Power Plants for Providing Multiple Market and Local Network Services," Electric Power Systems Research, 189, 2020, doi:10.1016/j.epsr.2020.106775.

[21] Levent Yavuz, Ahmet Önen, S.M. Muyeen and Innocent Kamwa, "Transformation of Microgrid to Virtual Power Plant - a Comprehensive Review," IET Generation Transmission \& Distribution, 13(11), 1994-2005, 2018, doi:10.1049/iet-gtd.2018.5649.

[22] R. Bertram, A. Schnettler, "A control model of virtual power plant with reactive power supply for small signal system stability studies,"_2017 IEEE ManchesterPowerTech, Manchester, 1-6, 2017 , doi:10.1109/PTC.2017.7980838.

[23] F. Muuß, G.A.N. Hemdan, M. Kurrat, D. Unger, B. Engel, "Dynamic virtual reactive power plant in active distribution networks," IEEE Eindhoven PowerTech (PowerTech, 697-702, 2015, doi:10.1109/PTC.2015.7232356.

[24] IEEE Standard Definitions for the Measurement of Electric Power Quantities Under Sinusoidal, Nonsinusoidal, Balanced, or Unbalanced Conditions, IEEE Std., 1459-2010. 